\title{
The Preliminary Study of Effects of Tolfenamic Acid on Cell Proliferation, Cell Apoptosis, and Intracellular Collagen Deposition in Keloid Fibroblasts In Vitro
}

\author{
Dan Yi, ${ }^{1}$ Ji Bihl, ${ }^{1}$ Mackenzie S. Newman, ${ }^{1}$ Yanfang Chen, ${ }^{1}$ and Richard Simman ${ }^{1,2}$ \\ ${ }^{1}$ Department of Pharmacology and Toxicology, Boonshoft School of Medicine, Wright State University, 3640 Colonel Glenn Hwy, \\ Dayton, OH 45435, USA \\ ${ }^{2}$ Department of Plastic and Reconstructive Surgery, Boonshoft School of Medicine, Wright State University, 3640 Colonel Glenn Hwy, \\ Dayton, $\mathrm{OH} 45435$, USA
}

Correspondence should be addressed to Yanfang Chen; yanfang.chen@wright.edu and Richard Simman; plasticsimman@yahoo.com

Received 30 May 2014; Revised 28 August 2014; Accepted 28 August 2014; Published 22 September 2014

Academic Editor: Lajos Kemény

Copyright (C) 2014 Dan Yi et al. This is an open access article distributed under the Creative Commons Attribution License, which permits unrestricted use, distribution, and reproduction in any medium, provided the original work is properly cited.

\begin{abstract}
Keloid scarring is a fibroproliferative disorder due to the accumulation of collagen type I. Tolfenamic acid (TA), a nonsteroidal anti-inflammatory drug, has been found to potentially affect the synthesis of collagen in rats. In this preliminary study, we aimed to test the effects of TA on cell proliferation, cell apoptosis, and the deposition of intracellular collagen in keloid fibroblasts. Normal fibroblasts (NFs) and keloid fibroblasts (KFs) were obtained from human dermis tissue. Within the dose range $10^{-3}-$ $10^{-6} \mathrm{M}$ and exposure times $24 \mathrm{~h}, 48 \mathrm{~h}$, and $72 \mathrm{~h}$, we found that $0.55 \times 10^{-3} \mathrm{M}$ TA at $48 \mathrm{~h}$ exposure exhibited significantly decreased cell proliferation in both NFs and KFs. Under these experimental conditions, we demonstrated that (1) TA treatment induced a remarkable apoptotic rate in KFs compared to NFs; (2) TA treatment reduced collagen production in KFs versus NFs; (3) TA treatment decreased collagen type I expression in KFs comparing to that of NFs. In summary, our data suggest that TA decreases cell proliferation, induces cell apoptosis, and inhibits collagen accumulation in KFs.
\end{abstract}

\section{Introduction}

Keloid scarring, is a raised scar which forms by expanding beyond the boundaries of the original lesion [1]. The main histological manifestation of a keloid scar is the overgrowth of atypical fibroblasts with excessive accumulation of extracellular matrix components, especially collagen, fibronectin, elastin, and proteoglycans [1-4]. The causes of this type of scar are still unknown, but it has been pointed out that keloid scars can develop after any dermal abrasion including burns, piercing, or surgery [1-6]. Differing from normal wound healing, keloid scar formation begins with abnormal tissue growth in the dermal lesion extending beyond the borders of the original wound [7-11]. The central pathological wound healing response of keloid scarring is composed of a high density of mesenchymal cells called keloid fibroblasts (KFs) $[9,10]$. Consequently, the over growth of KFs results in overabundance of extracellular and intracellular matrix stroma, which is classified by irregularly directed and thick hyalinized spiral bundles described as keloidal collagen [5, 12]. During the formation of keloid scars, the type of collagen initially secreted by fibroblasts is granular collagen type III. Throughout the maturation of the process, collagen type I gradually replaces collagen type III and eventually comprises extracellular matrix in 99\% of the wound bed [1, 5, 9-12]. Current, common treatments for keloids are often a combination of excision followed by a reconstructive surgical procedure. Glucocorticoids or 5-fluorouracil injections followed by compression therapy such as silicone sheets are frequently employed $[1,2]$. Nonetheless, recurrence remains between $45 \%$ and $100 \%[3,4]$. Therefore, treatment of keloids continues to be a great challenge for the reconstructive surgeon.

Tolfenamic acid (TA) is a fenamic acid derivative belonging to the nonsteroidal anti-inflammatory drug (NSAID) 
<smiles>Cc1c(Cl)cccc1Nc1ccccc1C(=O)O</smiles>

Figure 1: Structure of tolfenamic acid.

class that is traditionally used for rheumatic diseases $[13,14]$. The predominant medical uses of this group of drugs include rheumatoid arthritis, osteoarthritis, and inflammatory arthropathies [15-18]. TA, also written as 2([3-chloro-2-methylphenyl]-amino)-benzoic acid in IUPAC terms (Figure 1), has a low solubility in water and molecular weight of $261.7 \mathrm{~g} / \mathrm{mol}$. The exact medical applications, adverse effects, and mechanism of TA are not clear. However, previous studies have described highly specific applications of TA. Studies illustrate that TA is associated with inhibiting collagen metabolism in connective tissue in rats and has the capacity to induce cancer cell apoptosis [13, 14, 19-25]. There is an inhibition of sodium tolfenamate on the metabolism of collagen with $0.15 \mathrm{~mol} / \mathrm{L} \mathrm{NaCl}$ in rats [13]. TA reduces cell survival, growth, and angiogenesis in tumor and cancer cells, including human xenograft tumor, human pancreatic cancer, human neuroblastoma, and mouse prostate cancer by regulating the activity of transcription factor Spl; human head and neck cancer by regulating NADAG-1; human colorectal cancer via ESE-1/EGR-1; and human oral cancer by affecting the p38 mitogen-activated protein kinase signaling pathway [14, 19-23].

\section{Materials and Methods}

2.1. Cell Culture and Chemicals. All skin samples were obtained under Wright State University IRB number SC4833. A sample of scar tissue (KF1) was taken from a 24-yearold African-American male with clinical and pathologic evidence of keloid scarring confirmed as previously described $[10,26]$. A second keloid fibroblast cell line (KF2) was obtained from a 35-year-old African-American female was purchased from ATCC (Passage 11, ATCC, USA). One normal adult skin sample (NF) was obtained from a 29 -year-old African-American female during plastic surgery $[7,8]$. Skin specimens were incubated with $2 \mathrm{~mL}$ digestion medium containing high glucose Dulbecco's Modified Eagle's Medium (DMEM) (Gibco, Life Technologies, USA), $5 \mathrm{mg} / \mathrm{mL}$ collagenase/dispase II (RocheDiagnostics, USA), and $0.25 \%$ trypsin (Invitrogen, Life Technologies, USA) for 8 hours under 5\% $\mathrm{CO}_{2}$, at $37^{\circ} \mathrm{C}[7,9,27]$. Isolated fibroblasts passages (P) 0 from keloid scar tissue and normal dermis tissue were cultured in total medium comprising of high glucose DMEM, $10 \%$ fetal bovine serum (Gibco, Life Technologies, USA), 1\% pen/strep/glutamine (Invitrogen, Life Technologies, USA) in the condition of $5 \% \mathrm{CO}_{2}$, at $37^{\circ} \mathrm{C}$. Each cell line was cultured separately. KF1 P13 to P15, KF2 P 3 to 5, and NF P3-P8 were tested in all assays. As both KF1 and KF2 were conducted in each assay, generally it will refer to KF in the following. TA was purchased from Cayman Chemical Company, USA.
2.2. MTT. NFs and KFs were divided into different groups for pretreatment with TA $\left(10^{-3} \mathrm{M}, 10^{-4} \mathrm{M}, 10^{-5} \mathrm{M}\right.$, or $\left.10^{-6} \mathrm{M}\right)$ for different periods $(24 \mathrm{~h}, 48 \mathrm{~h}$, or $72 \mathrm{~h}$ ), respectively. An MTT kit (Invitrogen, Life Technologies, USA) was used for the proliferation assay. Absorbance was detected at $535 \mathrm{~nm}$ in a Packard Fusion spectrophotometer [27]. Relatives of Cell proliferation rate (\%) were determined with cell cultures and analyzed according to the following equation:

$$
\begin{aligned}
& \text { Fibroblast proliferation rate (\%) } \\
& =\frac{\text { OD535 (TA treatment group) }}{\text { OD535 (Non-TA treatment group) }}
\end{aligned}
$$

All experiments were performed independently. Six times with keloid fibroblast cultures culture as well as normal skin dermal culture.

2.3. Apoptosis. NFs and KFs were divided into three groups Control ("C", only treated with medium), Vehicle ("V", medium $0.55 \% \mathrm{DMSO})$, and TA $(550 \mu \mathrm{M}$ TA dissolved in $0.55 \%$ DMSO with medium) for $48 \mathrm{~h}$ exposure separately. Annexin-binding buffer (Invitrogen, Life Technologies, USA) and propidium iodide (Invitrogen, Life Technologies, USA) working solutions were prepared at $1 \mathrm{X}$. Cell apoptosis were detected by a fluorescence-activated cell sorting (FACS) Calibur Flow Cytometer (Accuri C6, Inc., USA) [20, 21]. All experiments were performed independently. Four times with keloid fibroblast cultures culture as well as normal skin dermal culture.

2.4. Collagen Staining. NFs and KFs were divided into three groups Control ("C", only treated with medium), Vehicle ("V", medium $0.55 \% \mathrm{DMSO})$, and TA $(550 \mu \mathrm{M}$ TA dissolved in $0.55 \%$ DMSO with medium) for $48 \mathrm{~h}$ exposure separately. Sirius red/Fast green staining kit (Chondrex, USA) was used to analyze collagen production. Absorbances at $535 \mathrm{~nm}$ and $600 \mathrm{~nm}$ were detected by a Packard Fusion spectrophotometer and analyzed by the following equation:

$$
\text { Collagen }(\mu \mathrm{g} / \text { well })=\frac{\text { OD535 }-0.291 * \text { OD600 }}{0.037} \text {. }
$$

All experiments were performed independently. Four times with keloid fibroblast cultures culture as well as normal skin dermal culture.

2.5. Western Blot. NFs and KFs were divided into three groups Control ("C", only treated with medium), Vehicle ("V", medium $0.55 \% \mathrm{DMSO})$, and TA $(550 \mu \mathrm{M}$ TA dissolved in $0.55 \%$ DMSO with medium) for $48 \mathrm{~h}$ exposure separately. Proteins were extracted from each cell line and the concentrations were measured by BCA assay. Sixty micrograms of each sample were mixed with $10 \mu \mathrm{L}$ of $5 \mathrm{X}$ protein sample loading buffer. The trans-blotted PVDF membrane was then blocked with blocking buffer (3\% BSA; IX TBS; and 0.05\% Tween-20) for $1 \mathrm{~h}$. Afterward, It was incubated with rabbit monoclonal anti-human collagen type I (dilution $1: 2000$, Thermo Fisher Scientific, USA), over two nights at $4^{\circ} \mathrm{C}$. 


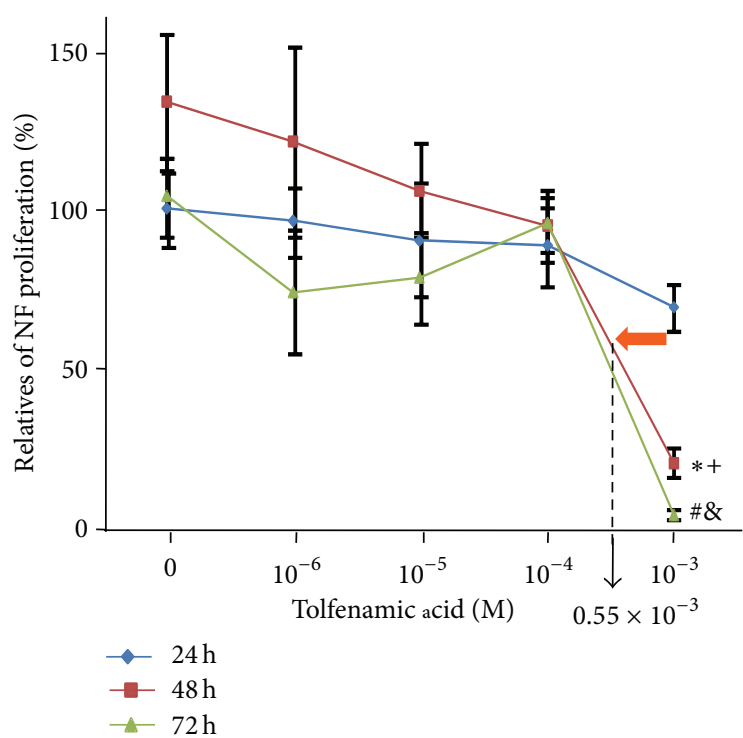

(a)

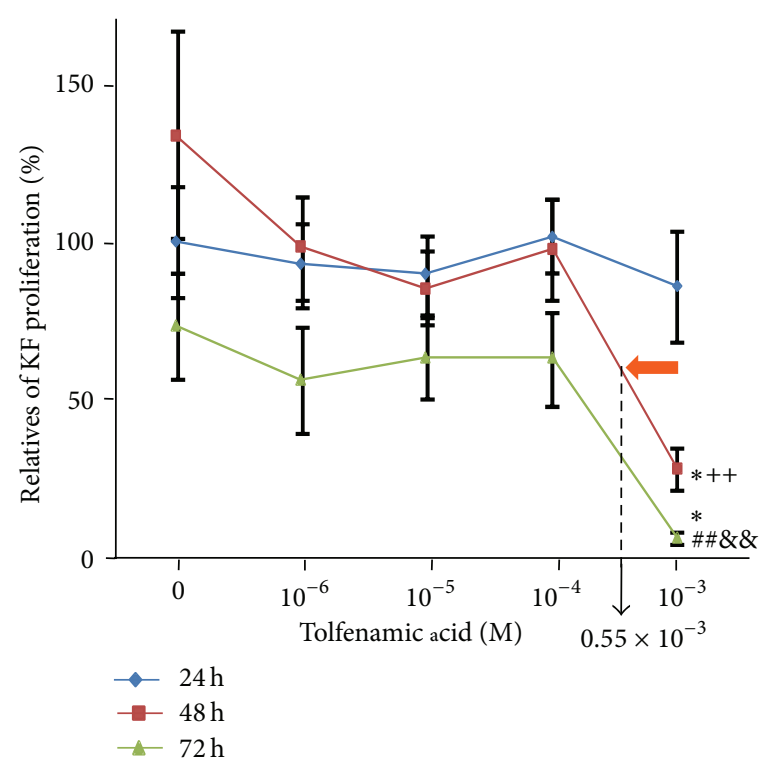

(b)

FIGURE 2: The dose- and time-responses of TA on cell proliferation in NFs and KFs. In $48 \mathrm{~h}$ exposure time group and $72 \mathrm{~h}$ exposure time group, $10^{-3} \mathrm{M}$ TA significantly decreased cell proliferation in NFs. $0,10^{-6}, 10^{-5}, 10^{-4}, 10^{-3}, 0.55 \times 10^{-3}$ : M TA was dissolved in $1 \%$ DMSO. ${ }^{*} \mathrm{P}<0.01,10^{-3} \mathrm{M}$ versus $0 \mathrm{M} ;{ }^{+} P<0.01,48 \mathrm{~h}$ versus $24 \mathrm{~h} ;{ }^{\#} \mathrm{P}<0.01,10^{-3} \mathrm{M}$ versus $0 \mathrm{M}$ or $10^{-4} \mathrm{M} ;{ }^{8} \mathrm{P}<0.01,72 \mathrm{~h}$ versus $24 \mathrm{~h}$ or $48 \mathrm{~h}$; ${ }^{* *} \mathrm{P}<0.01,10^{-3} \mathrm{M}$ versus $0 \mathrm{M} ;{ }^{++} \mathrm{P}<0.05,48 \mathrm{~h}$ versus $24 \mathrm{~h} ;{ }^{\# \#} \mathrm{P}<0.05,10^{-3} \mathrm{M}$ versus $0 \mathrm{M}$; ${ }^{\& \&} \mathrm{P}<0.01,72 \mathrm{~h}$ versus $24 \mathrm{~h}$ or $48 \mathrm{~h} ; n=6$.

Anti-rabbit HRP-conjugated secondary antibody (dilution at $1: 20000$, Sigma, USA) was then added to the membrane on a shaker for an hour at room temperature. After rinsing with TBST, the membrane was activated with chemiluminescent HRP substrate (Cell Signaling Technology, USA) for $4 \mathrm{~min}$ in the dark at room temperature. It was visualized and quantified using a chemiluminescent detection system (Bio-Rad, USA). Protein band intensity in each lane was scored by volume intensity and was normalized to beta-actin (dilution at 1:4000, Sigma, USA) [27]. All experiments were performed independently. Four times with keloid fibroblast cultures culture as well as normal skin dermal culture.

2.6. Statistics. Statistical analysis was performed with STATISTICA version 6.0 (StatSoft, Inc. USA). Data are displayed as mean \pm SEM which was evaluated by one-way ANOVA between two groups in this study. $P<0.05$ was considered statistically significant for all tests.

\section{Results}

3.1. Dose- and Time-Response of TA on NFs and KFs Cell Proliferation. The effect of TA on NFs and KFs proliferation was evaluated by MTT analysis and this is displayed in Figure 2. At the highest concentration of TA, cell proliferation rates appeared to decrease. As the exposure time increased, $\mathrm{NF}$ and KF proliferation sharply decreased at $10^{-3} \mathrm{M}$ TA. Cell proliferation also decreased at a greater rate when TA was applied over 48 or 72 hours. Based on the results of this assay, $0.55 \times 10^{-3} \mathrm{M}$ TA at $48 \mathrm{~h}$ exposure was determined to be the ideal condition for future experimentation.
3.2. The Effect Dose and Time of TA on Inducing Cell Apoptosis in KFs. To determine whether TA induced cell apoptosis on NFs and KFs, Annexin-V/PI labeling was administered after applying $0.55 \times 10^{-3} \mathrm{M}$ TA dissolved in $0.55 \%$ DMSO for $48 \mathrm{~h}$. Although the vehicle alone exhibited an effect on NFs, selectively significant apoptosis in TA-treated KFs compared with non-TA treatment groups of KFs and in NFs (Figure 3).

\subsection{The Effect Dose and Time of TA on Inhibiting Collagen} Accumulation in KFs. Quantitative analysis of intracellular and extracellular Sirius red fluorescent staining reflects the degree of all types of collagen produced by fibroblasts. Results indicated that collagen production was significantly reduced in KFs at the previously determined dose and time (Figure 4). Additionally, there was a considerable morphological change in KFs. Stained intensity decreased in all samples. Secreted, stained proteins and cell density were both reduced, especially in KF cultures.

3.4. The Effect Dose and Time of TA on Reducing the Expression of Collagen Type I in KFs. The effects of TA on collagen type I (Collagen I) protein expression were evaluated by Western blot in both cell types. Protein quantity was measured 48 hours after each treatment. Figure 5 shows a strong increased expression in KFs compared to NFs. TA significantly suppressed the expression of collagen type I in KFs compared with the other groups, while the inhibition of collagen type I expression in NFs after TA treatment was not significant. This result demonstrated that $0.55 \times 10^{-3} \mathrm{M}$ TA for $48 \mathrm{~h}$ inhibits collagen I expression significantly in KFs but not, effectively in NFs. 
C

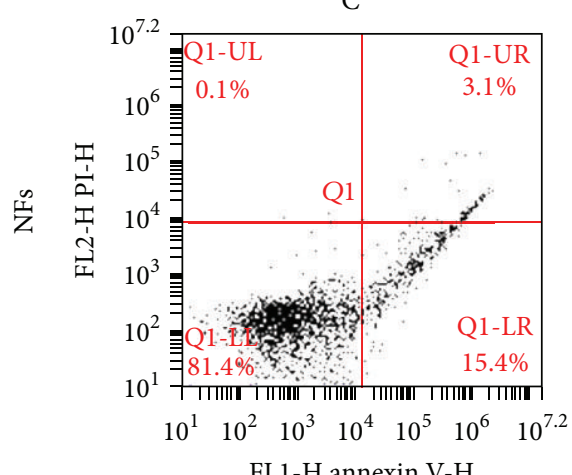

FL1-H annexin V-H

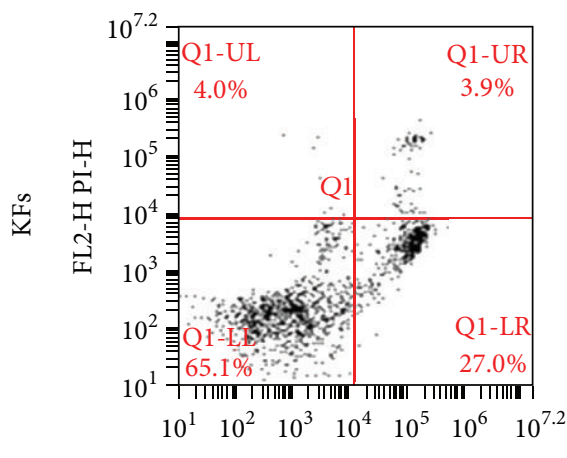

FL1-H annexin V-H
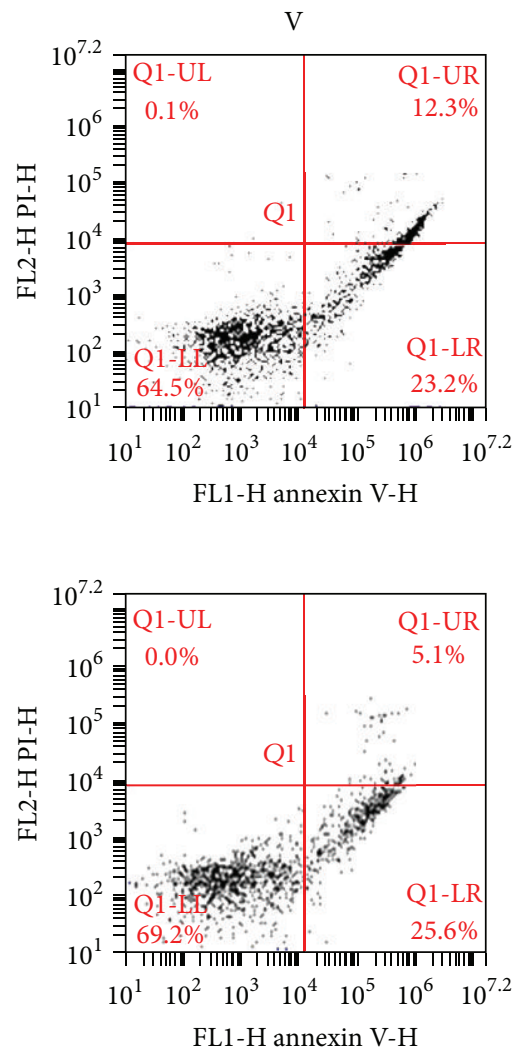

FL1-H annexin V-H

(a)
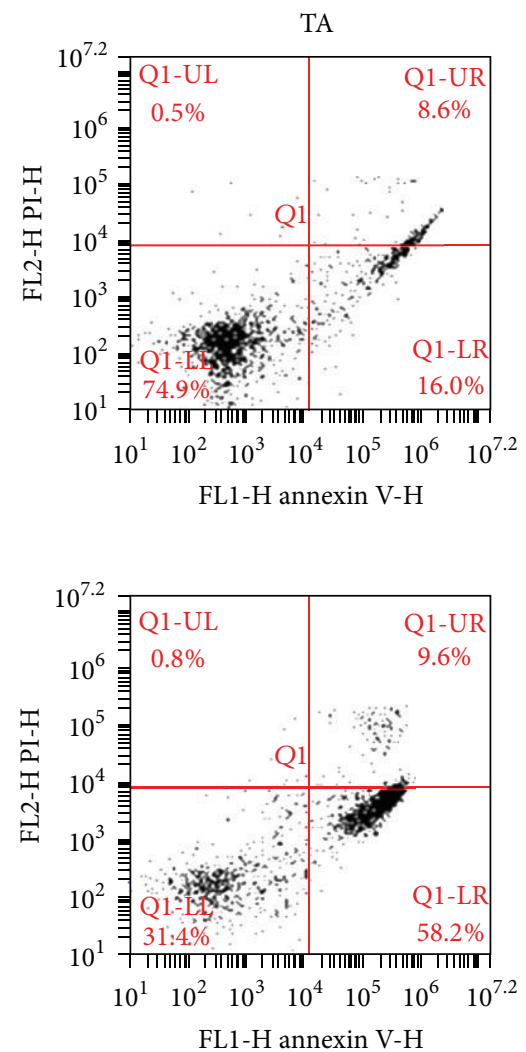

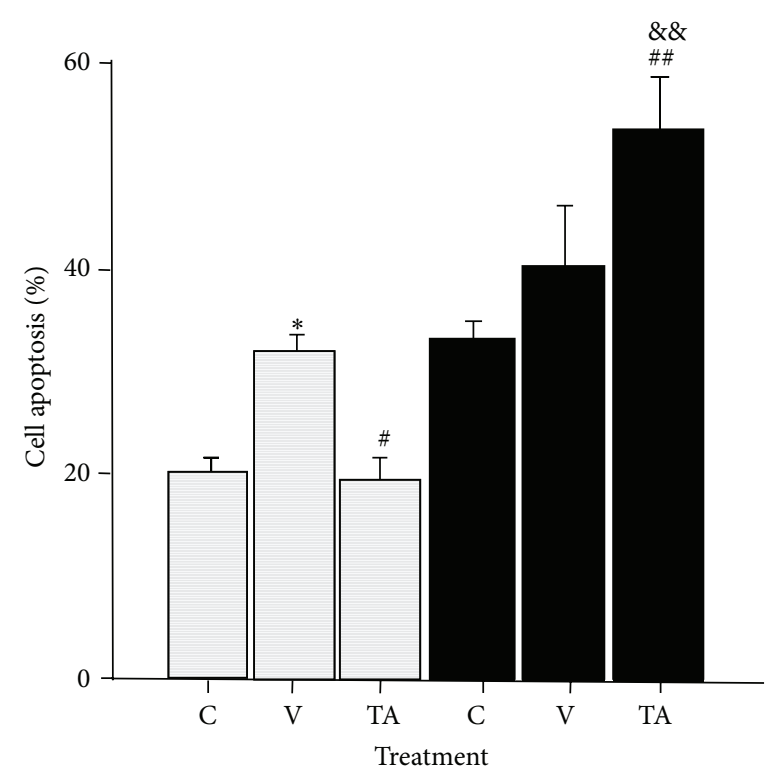

目 NFs

(b)

FIGURE 3: The effect of TA on cell apoptosis in NFs and KFs. DMSO induced early and late apoptosis in NFs and slightly induced early apoptosis in KFs. TA strikingly induced late apoptosis in KFs. C: control, total medium; V: vehicle, $0.55 \%$ DMSO; TA, $0.55 \times 10^{-3} \mathrm{M}$ of TA was dissolved in $0.55 \%$ DMSO. ${ }^{*} P<0.01$, V versus $\mathrm{C}$ or TA; ${ }^{\#} P<0.05$, TA versus $\mathrm{V}$; ${ }^{\# \#} P<0.05$, TA versus $\mathrm{C}$; ${ }^{\text {\&\& }} P<0.01$, TA versus TA; $n=6$. 

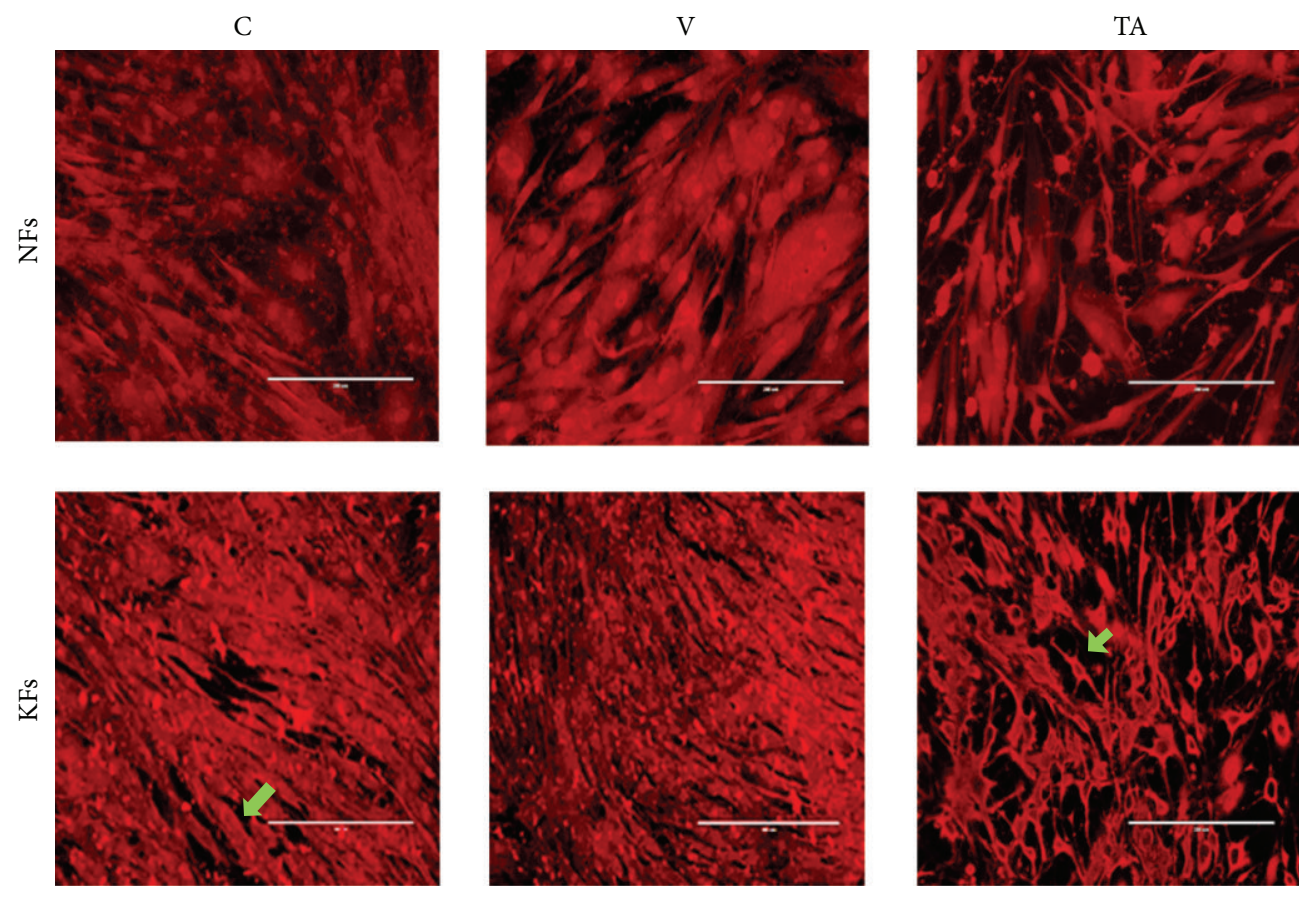

(a)

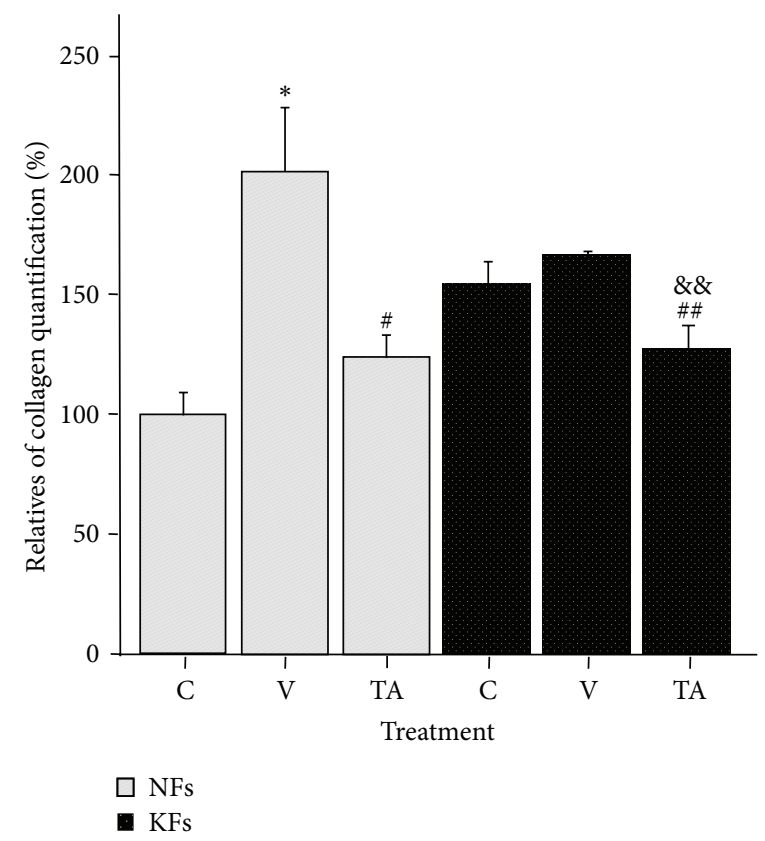

(b)

FIGURE 4: The effect of TA on collagen production in NF and KF after Sirius red staining. DMSO significantly increased collagen production in NFs. TA strikingly reduced collagen production in KFs compared with NFs. C: control, total medium; V: vehicle, $0.55 \%$ DMSO; TA, $0.55 \times$ $10^{-3} \mathrm{M}$ of TA was dissolved in $0.55 \%$ DMSO. ${ }^{*} P<0.01$, V versus $C ;{ }^{\#} P<0.05$, TA versus $\mathrm{V} ;{ }^{\# \#} P<0.05$, TA versus $C$; ${ }^{\& \&} P<0.01$, TA versus $\mathrm{V} ; n=4$.

\section{Discussion}

Keloid scaring, a type of scars, is a fibroproliferation disease with the accumulation of collagen deposition caused by the upregulation of autocrine TGF- $\beta$ signaling during the wound healing process $[5,13,27]$. The role of TA, a fenamate and NASID, has been used recently for cancer treatments $[13,14,19-25]$. These experiments were designed to determine if the administration of TA is able to alter the proliferatory rates of keloid fibroblasts and decrease collagen production in KFs. Our preliminary study demonstrates that TA has the potential to normalize some of the characteristic 


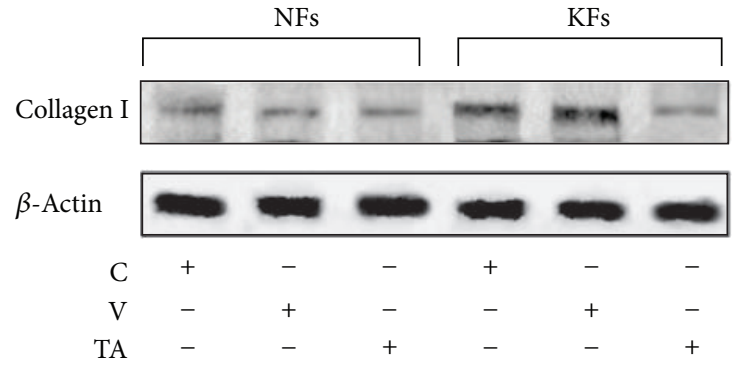

(a)

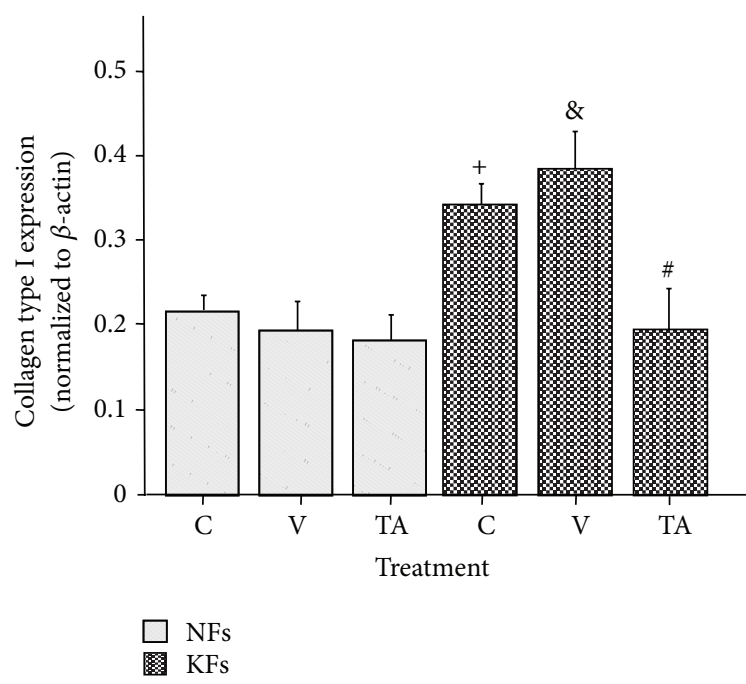

(b)

FIgURE 5: The effect of TA on the expression of collagen type I in NFs and KFs. DMSO slightly increased the expression of collagen type I in KFs. TA significantly inhibited the expression of collagen type I in KFs. C: control, total medium; V: vehicle, $0.55 \% \mathrm{DMSO}$; TA, $0.55 \times 10^{-3} \mathrm{M}$ of TA was dissolved in $0.55 \%$ DMSO ${ }^{+} P<0.05$, C versus $C$; ${ }^{\&} P<0.05$, V versus NFs $C$; ${ }^{\#} P<0.05$, TA versus V; $n=4$.

features of KFs such as cell apoptosis and collagen production.

Initially, we tested the effects of TA on NF and KF proliferation by applying five concentrations of TA $\left(10^{-3} \mathrm{M}\right.$, $10^{-4} \mathrm{M}, 10^{-5} \mathrm{M}$, and $10^{-6} \mathrm{M}$ ) for 3 different exposure times ( $24 \mathrm{~h}, 48 \mathrm{~h}$, and $72 \mathrm{~h}$ ) separately in order to determine the conditions at which minimal drug concentration had the greatest proportionate effect on disease fibroblasts. We found that NF and KF proliferation rates were decreased with the $10^{-3} \mathrm{M}$ TA after $48 \mathrm{~h}$ exposure. This indicated that the diversity of NF and KF proliferation were significantly reduced by certain higher amount of TA. Linear extrapolation of cell proliferation assay, data at $48 \mathrm{~h}$ revealed that the effective concentration of TA which can result in half reduction of NF proliferation was $550 \mu \mathrm{M}$. This concentration of TA at $48 \mathrm{~h}$ exposure did not show a significant reduction in KFs proliferation, but other effects were still expected to be present. At 72 hours, there was a large reduction in proliferation of KF cultures versus NFs. This may be due to a long-phase response to TA toxicity in KF or a side effect of changing culture media every three days. To further qualify the effects of this TA does and time exposure treatment, we conducted cell apoptosis assays with $0.55 \times 10^{-3} \mathrm{M}$ TA and with $48 \mathrm{~h}$ time exposure. A group exposed to $0.55 \%$ DMSO in medium was added to control for vehicle effects. Though $0.55 \%$ DMSO induced a proportional apoptotic rate in NFs and KFs, cell apoptosis in KFs after TA treatment induced a selectively significant apoptotic rate compared to NFs. This indicated that cell apoptosis was significantly influenced by TA. According to the typical histological changes in KFs [1-4], reducing collagen production is a primary pharmacological target to treat keloid scarring. In our study, we tested the effects of TA on decreasing the accumulation of collagen and furthermore tried to verify a relationship between cell apoptosis and collagen deposition. Based on our data, a high inhibition rate of collagen production was detected in KFs after $0.55 \times 10^{-3} \mathrm{M}$ TA with $48 \mathrm{~h}$ exposure time compared with NFs. Although the effect of $0.55 \%$ DMSO increased collagen production in NFs, the production caused by $0.55 \%$ DMSO did not show specific significance in KFs. Concurrently, after TA treatment, cell number also decreased. Therefore, TA reduced collagen production in KFs with high efficiency. The Sirius red collagen quantification assay targets the helical collagen repeat bundles and is therefore nonspecific for specific types of collagens. The majority of collagen produced by KFs is collagen type I, and our results were specific for collagen type I after TA treatment $[1,2,12,26-28]$. Our study confirms that collagen I is overexpressed in KFs versus NFs. Treatment with TA in vitro significantly decreased collagen expression in KFs over NFs.

In the cell apoptosis and collagen expression experiments, DMSO vehicle alone did have some effects compared to control group. The underlying mechanism for this is not apparent from our study. In cell apoptosis assays, compared with the treatment group, the vehicle group had more significant effect in NFs while the effect in KFs is not significant. This may be further considered for clinical studies. In collagen expression assays, DMSO did not significantly induce collagen expression in NFs. In KFs, it significantly increased the expression of collagen but this effect can be reversed by TA. In summary, DMSO did not influence the performance of TA on cell apoptosis and collagen expression in KFs. The present study is limited by the number of samples. Nonetheless, statistically significant effects were seen from TA treatment in KF cells. A potential mechanism for the activity of TA in keloid is its capacity to induce degradation of the Sp2 transcription factor. Sp1 has a role in the canonical TGF- $\beta$ transduction pathway. Because TGF- $\beta$ is widely regarded 
as the central progenitor of fibrotic scars and has a large capacity for autocrine signaling, the degradation of Sp1 may serve as a putative pharmacological target in keloids. Future studies may include the use of TGF- $\beta$ receptor blockers and measuring intracellular phosphorylated SMAD family members. Other studies may include work to refine the dosage with respect to solubility without a vehicle. Although a genetic mouse model does not yet exist for keloids, there are still valid models of keloid and hypertrophic scarring that may function to validate the mechanism and activity of TA in this disease. Despite the shortcomings of this preliminary study, TA has clear potential to selectively treat keloid fibroblasts over normal dermal fibroblasts. Numerous other small-molecule treatments have been tested in keloids, but TA and fenamates on the whole, have not been explored.

Generally speaking, our current novel data demonstrated that tolfenamic acid induced cell apoptosis and inhibited collagen production in keloid fibroblasts. TA could be the new therapeutical application for treating keloid scars. With the development of the advanced technologies, hundreds and thousands of treatments on keloid scar have been reported. However, this is the first time where TA was successfully used in vitro to induce cell apoptosis and reduce collagen accumulation in KFs. Furthermore, TA is an available commercial formulation chemical. Thereby, TA is recommended for clinical trials to confirm our findings.

\section{Conflict of Interests}

The authors declare that there is no conflict of interests regarding the publication of this paper.

\section{References}

[1] C. Chipev, R. Simman, G. Hatch, A. E. Katz, D. M. Siegel, and M. Simon, "Myofibroblast phenotype and apoptosis in keloid and palmar fibroblasts in vitro," Cell Death and Differentiation, vol. 7, no. 2, pp. 166-176, 2000.

[2] R. Simman, H. Alani, and F. Williams, "Effect of mitomycin C on keloid fibroblasts: an in vitro study," Annals of Plastic Surgery, vol. 50, no. 1, pp. 71-76, 2003.

[3] C. H. Ricketts, L. Martin, D. T. Faria, G. M. Saed, and D. P. Fivenson, "Cytokine mRNA changes during the treatment of hypertrophic scars with silicone and nonsilicone gel dressings," Dermatologic Surgery, vol. 22, no. 11, pp. 955-959, 1996.

[4] J. Meenakshi, V. Jayaraman, K. M. Ramakrishnan, and M. Babu, "Ultrastructural differentiation of abnormal scars," Annals of Burns and Fire Disasters, vol. 18, pp. 83-88, 2005.

[5] H. Wang and S. Luo, "Establishment of an animal model for human keloid scars using tissue engineering method," Journal of Burn Care and Research, vol. 34, no. 4, pp. 439-446, 2013.

[6] A. Asilian, A. Darougheh, and F. Shariati, "New combination of triamcinolone, 5-fluorouracil, and pulsed-dye laser for treatment of keloid and hypertrophic scars," Dermatologic Surgery, vol. 32, no. 7, pp. 907-915, 2006.

[7] C.-C. E. Lan, I.-H. Liu, A.-H. Fang, C.-H. Wen, and C.-S. Wu, "Hyperglycaemic conditions decrease cultured keratinocyte mobility: implications for impaired wound healing in patients with diabetes," The British Journal of Dermatology, vol. 159, no. 5, pp. 1103-1115, 2008.
[8] T. T. Phan, I. J. Lim, O. Aalami et al., "Smad3 signalling plays an important role in keloid pathogenesis via epithelialmesenchymal interactions," Journal of Pathology, vol. 207, no. 2, pp. 232-242, 2005.

[9] Y. Yagi, E. Muroga, M. Naitoh et al., "An ex vivo model employing keloid-derived cell-seeded collagen sponges for therapy development," Journal of Investigative Dermatology, vol. 133, no. 2, pp. 386-393, 2013.

[10] F. Syed, D. Sherris, R. Paus, S. Varmeh, P. P. Pandolfi, and A. Bayat, "Keloid disease can be inhibited by antagonizing excessive mTOR signaling with a novel dual TORC1/2 inhibitor," The American Journal of Pathology, vol. 181, no. 5, pp. 1642-1658, 2012.

[11] F. Syed and A. Bayat, "Notch signaling pathway in keloid disease: enhanced fibroblast activity in a Jagged-1 peptidedependent manner in lesional vs. extralesional fibroblasts," Wound Repair and Regeneration, vol. 20, no. 5, pp. 688-706, 2012.

[12] W. J. Lee, I.-K. Choi, J. H. Lee et al., "Relaxin-expressing adenovirus decreases collagen synthesis and up-regulates matrix metalloproteinase expression in keloid fibroblasts: in vitro experiments," Plastic and Reconstructive Surgery, vol. 130, no. 3, pp. 407e-417e, 2012.

[13] J. Krajickova, V. Pesakova, M. Adam, and K. E. Senius, "Effect of tolfenamic acid on the metabolism of the main connective tissue components in rats," Arzneimittel-Forschung, vol. 37, no. 2, pp. 177-180, 1987.

[14] J.-H. Kim, J.-Y. Jung, J.-H. Shim et al., "Apoptotic effect of tolfenamic acid in KB human oral cancer cells: possible involvement of the p38 MAPK pathway," Journal of Clinical Biochemistry and Nutrition, vol. 47, no. 1, pp. 74-80, 2010.

[15] P. D. Gotzsche, "Meta-analysis of grip strength: most common, but superfluous variable in comparative NSAID trials," Danish Medical Bulletin, vol. 36, no. 5, pp. 493-495, 1989.

[16] P. C. Gotzsche, "Review of dose-response studies of NSAIDs in rheumatoid arthritis," Danish Medical Bulletin, vol. 36, no. 4, pp. 395-399, 1989.

[17] P. C. Gotzsche, "Methodology and overt and hidden bias in reports of 196 double-blind trials of nonsteroidal antiinflammatory drugs in rheumatoid arthritis," Controlled Clinical Trials, vol. 10, no. 1, pp. 31-56, 1989.

[18] S. Rossi, W. Ou, D. Tang et al., "Gastrointestinal stromal tumours overexpress fatty acid synthase," Journal of Pathology, vol. 209, no. 3, pp. 369-375, 2006.

[19] E.-S. Choi, J.-H. Shim, J.-Y. Jung et al., "Apoptotic effect of tolfenamic acid in androgen receptor-independent prostate cancer cell and xenograft tumor through specificity protein 1 ," Cancer Science, vol. 102, no. 4, pp. 742-748, 2011.

[20] S. U. Kang, Y. S. Shin, H. S. Hwang, S. J. Baek, S.-H. Lee, and C.-H. Kim, "Tolfenamic acid induces apoptosis and growth inhibition in head and neck cancer: involvement of NAG-1 expression," PLoS ONE, vol. 7, no. 4, Article ID e34988, 2012.

[21] D. Eslin, U. T. Sankpal, C. Lee et al., "Tolfenamic acid inhibits neuroblastoma cell proliferation and induces apoptosis: a novel therapeutic agent for neuroblastoma," Molecular Carcinogenesis, vol. 52, no. 5, pp. 377-386, 2013.

[22] M. Abdelrahim, C. H. Baker, J. L. Abbruzzese, and S. Safe, "Tolfenamic acid and pancreatic cancer growth, angiogenesis, and Sp protein degradation," Journal of the National Cancer Institute, vol. 98, no. 12, pp. 855-868, 2006.

[23] S.-H. Lee, H. B. Jae, K. C. Chang et al., "ESE-1/EGR-1 pathway plays a role in tolfenamic acid-induced apoptosis in colorectal 
cancer cells," Molecular Cancer Therapeutics, vol. 7, no. 12, pp. 3739-3750, 2008.

[24] M. Abdelrahim, C. H. Baker, J. L. Abbruzzese et al., "Regulation of vascular endothelial growth factor receptor-1 expression by specificity proteins 1,3 , and 4 in pancreatic cancer cells," Cancer Research, vol. 67, no. 7, pp. 3286-3294, 2007.

[25] U. T. Sankpal, M. Abdelrahim, S. F. Connelly et al., "Small molecule tolfenamic acid inhibits PC-3 cell proliferation and invasion in vitro, and tumor growth in orthotopic mouse model for prostate cancer," Prostate, vol. 72, no. 15, pp. 1648-1658, 2012.

[26] D.-L. Fan, W.-J. Zhao, Y.-X. Wang, S.-Y. Han, and S. Guo, "Oxymatrine inhibits collagen synthesis in keloid fibroblasts via inhibition of transforming growth factor- $\beta 1 /$ Smad signaling pathway," International Journal of Dermatology, vol. 51, no. 4, pp. 463-472, 2012.

[27] C. K. Lim, A. S. Halim, N. S. Yaacob, I. Zainol, and K. Noorsal, "Keloid pathogenesis via Drosophila similar to mothers against decapentaplegic (SMAD) signaling in a primary epithelialmesenchymal in vitro model treated with biomedical-grade chitosan porous skin regenerating template," Journal of Bioscience and Bioengineering, vol. 115, no. 4, pp. 453-458, 2013.

[28] C.-S. Wu, P.-H. Wu, A.-H. Fang, and C.-C. E. Lan, "FK506 inhibits the enhancing effects of transforming growth factor (TGF)- $\beta 1$ on collagen expression and TGF- $\beta /$ Smad signalling in keloid fibroblasts: implication for new therapeutic approach," The British Journal of Dermatology, vol. 167, no. 3, pp. 532-541, 2012. 


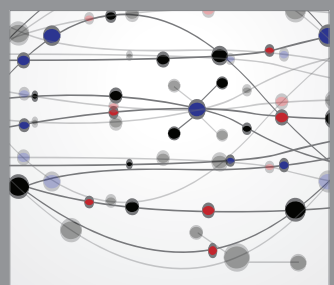

The Scientific World Journal
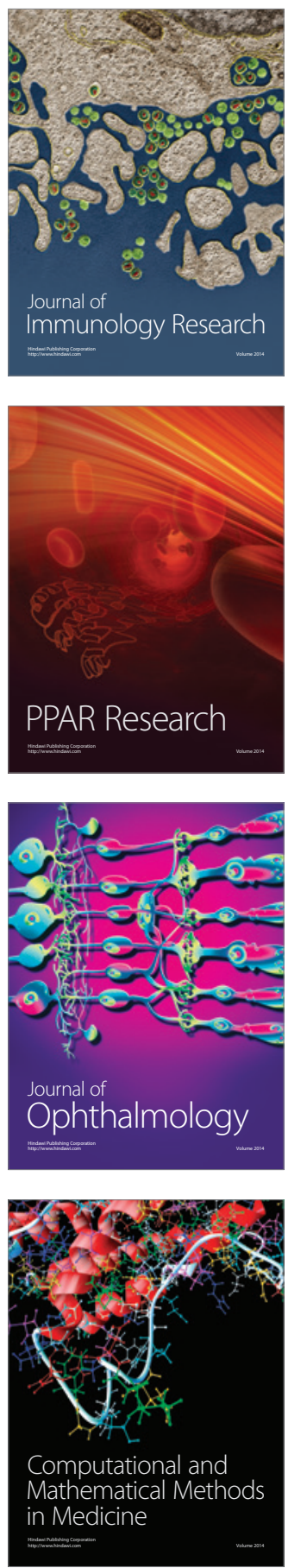

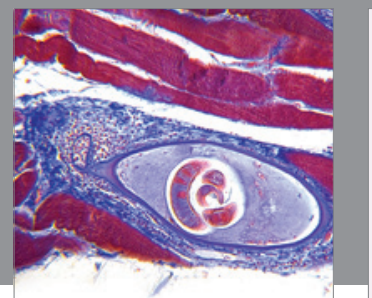

Gastroenterology

Research and Practice
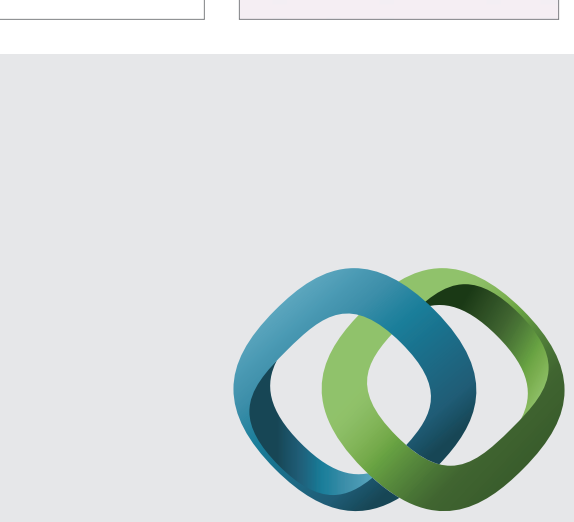

\section{Hindawi}

Submit your manuscripts at

http://www.hindawi.com
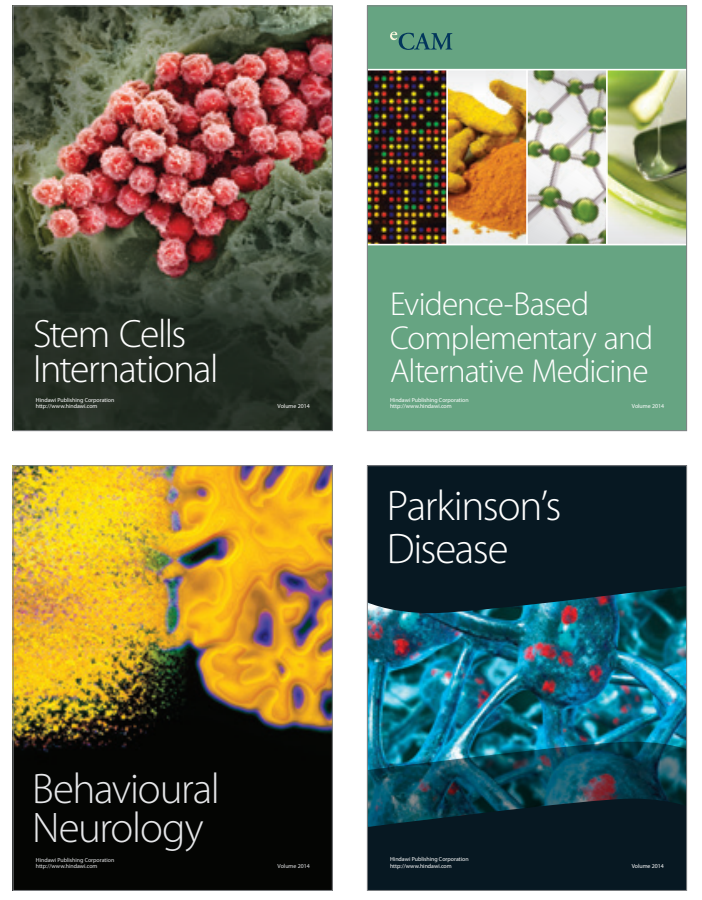
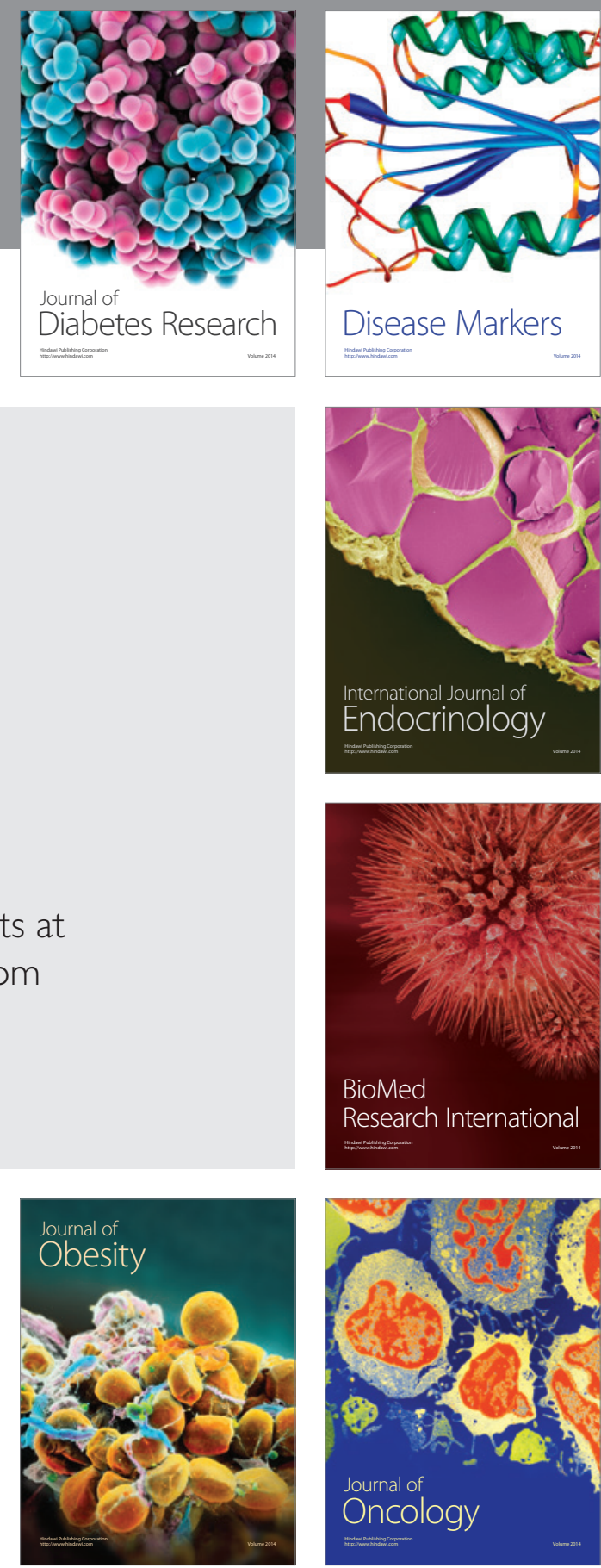

Disease Markers
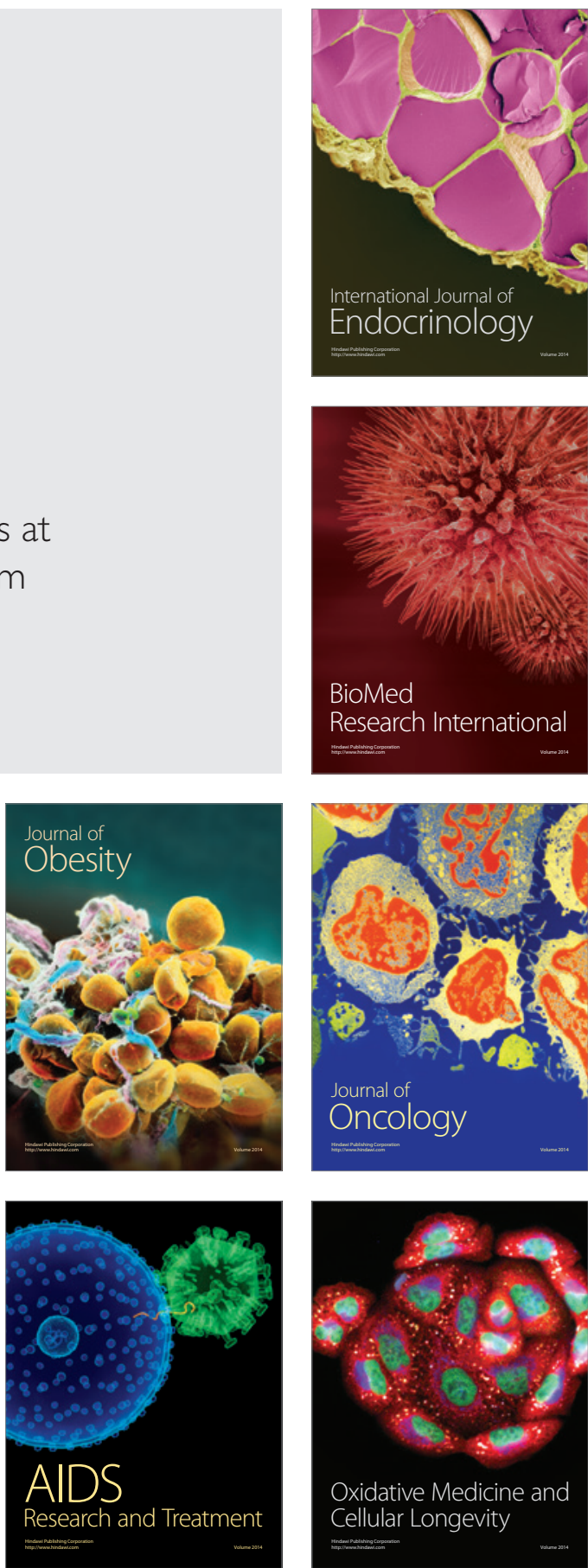